\title{
Orion Entry Performance-Based Center-of-Gravity Box
}

\author{
Jeremy R. Rea ${ }^{1}$ \\ NASA Johnson Space Center, Houston, TX, 77058
}

\begin{abstract}
The Orion capsule is designed both for Low Earth Orbit missions to the ISS and for missions to the moon. For ISS class missions, the capsule will use an Apollo-style direct entry. For lunar return missions, depending on the timing of the mission, the capsule could perform a direct entry or a skip entry of up to $4800 \mathrm{n}$.mi. in order to land in the coastal waters of California.
\end{abstract}

The physics of atmospheric re-entry determine the capability of the Orion vehicle. For a given vehicle mass and shape, physics tells us that the driving parameters for an entry vehicle are the hypersonic lift-to-drag ratio $(\mathrm{L} / \mathrm{D})$ and the flight path angle at entry interface $\left(\gamma_{\mathrm{EI}}\right)$. The design of the Orion atmospheric re-entry must meet constraints during both nominal and dispersed flight conditions on landing accuracy, heating rate, total heat load, sensed acceleration, and proper disposal of the Service Module. These constraints define an entry corridor in the space of $L / D-\gamma_{E I}$; if the vehicle falls within this corridor, then all constraints are met. The $\gamma_{\mathrm{EI}}$ dimension of the corridor can be further constrained by the gloads experienced during emergency entries. Thus, the entry performance for the Orion vehicle can be described completely by the $L / D$.

Bounds on the hypersonic $L / D$ necessary to achieve all the mission requirements can be defined for the given entry corridor. Landing accuracy performance drives the lower limit on $L / D$. In order to achieve the desired landing accuracy, a minimum $L / D$ must be ensured. The design of the Thermal Protection System (TPS) drives the upper limit on L/D. A higher L/D can drive mass into the design of the TPS. Conversely, once the TPS is designed, the L/D must be ensured to stay below a certain limit in order for the TPS to stay within its design envelop.

The L/D must stay within its upper and lower bounds during dispersed flight conditions. L/D is a function of both the aerodynamics and the center-of-gravity (CG) of the vehicle. The aerodynamics of the vehicle are determined by Computational Fluid Mechanics (CFD) and wind tunnel tests. However, the aerodynamics are not known precisely. Instead, an aerodynamic database has been developed where the aerodynamic coefficients are known to fall within a probabilistic band defined by upper and lower bounds. It is expected that the probabilistic band will shrink after the first missions are flown and real-world data is collected. Until that time, the Orion must be designed to the current aerodynamic database. Thus, for a given aerodynamic database with given uncertainties, the allowable range in $L / D$ can be mapped to an allowable box for the CG location. The CG box is used to set requirements on the dispersions allowed for vehicle packaging and cargo storage. As the aerodynamic uncertainties decrease, the size of the CG box can increase. This paper discusses the technique used to map the minimum and maximum $L / D$ bounds set by the entry performance requirements to the allowable dispersions in CG while accounting for aerodynamic uncertainties.

The $L / D$ is defined as the ratio of the lift force to the drag force. It is equivalent to the ratio of lift coefficient $\left(C_{L}\right)$ over drag coefficient $\left(C_{D}\right)$. $C_{L}$ and $C_{D}$ are functions of Mach number (M) and angle of attack $(\alpha)$. A Mach number of 25 is used as a measuring point of the hypersonic $L / D$. Variations in $C_{L}, C_{D}$, and $\alpha$ cause variations in $L / D$. Equation (1) shows the three contributions to the variation in $\mathrm{L} / \mathrm{D}$.

${ }^{1}$ Engineer, Flight Mechanics and Trajectory Design Branch, JSC-EG5, AIAA Member 


$$
\delta\left(\frac{L}{D}\right)=\left[-\frac{C_{L}}{C_{D}{ }^{2}}\right] \delta C_{D}+\left[\frac{1}{C_{L}}\right] \delta C_{L}+\left[\frac{\partial(L / D)}{\partial \alpha}\right] \delta \alpha
$$

The maximum variations in $C_{L}$ and $C_{D}$ are given in the aerodynamic database. The trim angle of attack $\left(\alpha_{\text {trim }}\right)$ is defined when the pitching moment coefficient is zero. The pitching moment coefficient $\left(C_{m}\right)$ is related to $\alpha$, the $X$-component of $C G\left(X_{C G}\right)$, and the $Z$ component of $\mathrm{CG}\left(\mathrm{Z}_{\mathrm{CG}}\right)$ by equation (2).

$$
\begin{aligned}
& C_{m}=C_{m_{o}}(M, \alpha)+C_{N}(M, \alpha) \frac{\Delta X_{C G}}{L_{r e f}}-C_{A}(M, \alpha) \frac{\Delta Z_{C G}}{L_{r e f}} \\
& \text { where: } \quad \begin{aligned}
C_{m} & =\text { Pitching coefficient about CG } \\
C_{m_{o}} & =\text { Pitching coefficient about MRC } \\
C_{N} & =\text { Normal force coefficient } \\
C_{A} & =\text { Axial force coefficient } \\
\Delta X_{C G} & =\left(X_{C G}-X_{M R C}\right) \\
\Delta Z_{C G} & =\left(Z_{C G}-Z_{M R C}\right) \\
L_{r e f} & =\text { Refernce length }
\end{aligned}
\end{aligned}
$$

Variations in $C_{m}, X_{C G}$, and $Z_{C G}$ cause variations in $\alpha_{\text {trim }}$. Equation (3) shows the contributions to the variation in $\alpha_{\text {trim }}$. It can be shown that $C_{m}$ and $Z_{C G}$ uncertainties are the major contributors to $L / D$ variation.

$$
\delta \alpha_{\text {trim }}=\frac{1}{C_{m_{\alpha}}}\left[\delta C_{m_{o}}-\frac{C_{A}}{L_{r e f}} \delta Z_{C G}+\frac{C_{N}}{L_{\text {ref }}} \delta X_{C G}\right]
$$

For a given Mach number, a desired $L / D$ value can be achieved with a specific value of $\alpha_{\text {trim. }}$ From equation (2), when Mach and $\alpha_{\text {trim }}$ are set, this defines a linear relationship between the $X_{C G}$ and $Z_{C G}$. Thus, a line of constant $L / D$ can be drawn on the vehicle in the XZ-plane. If the CG lies on this line, then the desired L/D will be achieved. When aerodynamic uncertainties are considered, the line of constant $L / D$ expands into a probability band of constant $\mathrm{L} / \mathrm{D}$. If the $\mathrm{CG}$ falls within this probability band, then there is a chance that the desired $L / D$ will be achieved.

Figure (1) below shows a notional example of the $L / D$ probability bands. The $X$-axis represents the $X_{C G}$ location, and the $Y$-axis represents the $Z_{C G}$ location. The blue band shows the region where it is possible to achieve the minimum $L / D$ for entry landing accuracy performance. The red band shows the region where it is possible to achieve the maximum L/D for entry TPS performance. The lines inside each band represent contours of probability. Each line represents a $5 \%$ increase in the chance that the $\mathrm{L} / \mathrm{D}$ will be outside its maximum or minimum bound. The dotted black line shows a notional nominal L/D line for nominal aerodynamics with no uncertainties. On this line is shown a typical CG dispersion. Each concentric ellipse represents a Gaussian distribution on CG at a level of 1-sigma, 2sigma, or 3-sigma. It can be seen that for this example box, there is a slight chance that the $\mathrm{L} / \mathrm{D}$ can be below the minimum value. In order to build robustness into the vehicle design, it is desired to limit the 3-sigma CG dispersions to keep them from intersecting the upper and lower $\mathrm{L} / \mathrm{D}$ probability bands. 


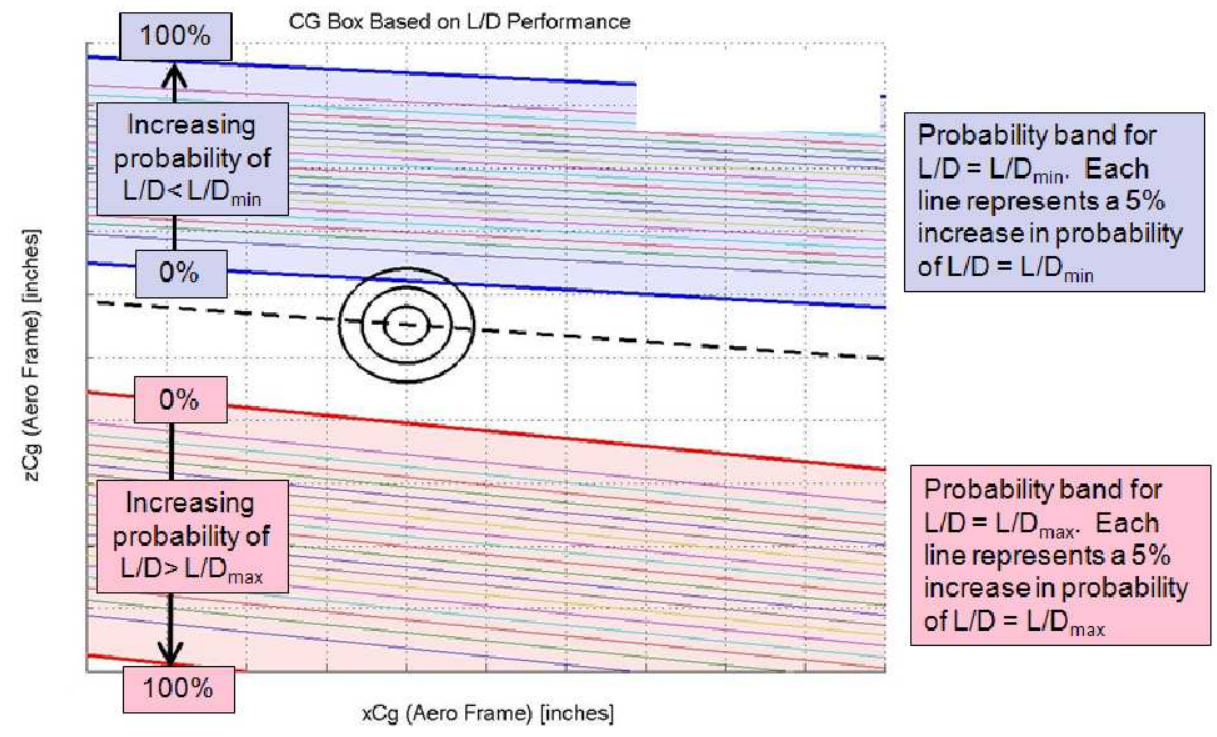

Figure (1) - Notional CG Box Based on L/D Performance

This figure is a graphical representation of the methodology used to define the allowable CG dispersions for the Orion vehicle. It can be seen that care must be taken to choose both the mean and 3-sigma CG values in order to meet the entry performance requirements. 